einzelnen Parlamentssitzungen. Bei den Beilagenbänden werden weniger wichtige Dokumente häufig zusammengefasst, wichtigere hingegen durch Sprungmarken hervorgehoben. So kann man z.B. die Texte der Aufbaugesetze Baden-Württembergs in den 1950er Jahren gezielt ansteuern oder durch den Inhalt der von den Parteien eingebrachten Anträge einen Eindruck von deren besonderen Anliegen gewinnen.

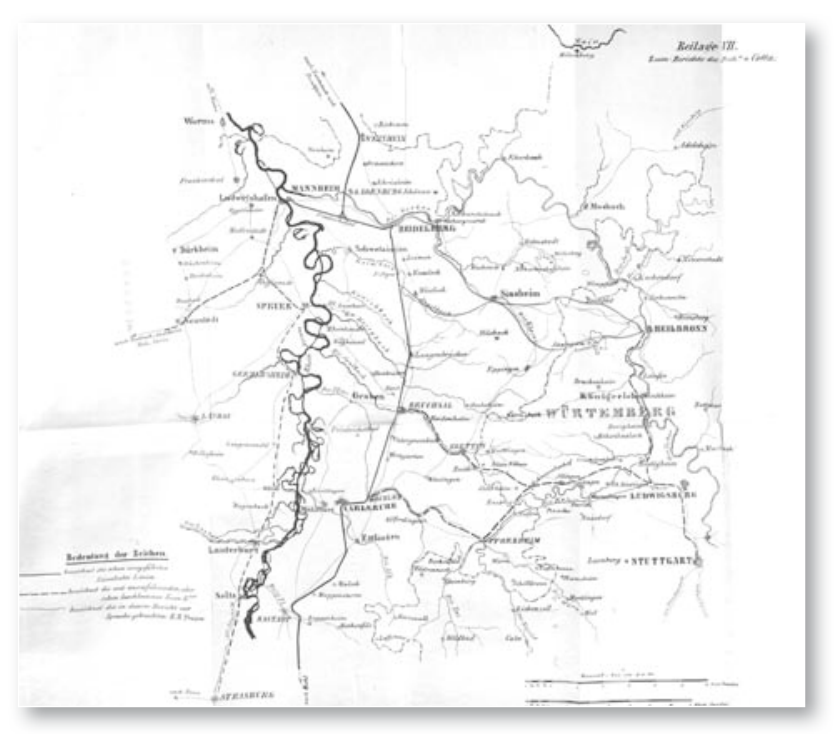

Wer Informationen zu sehr speziellen Themen braucht, sucht nach Hinweisen in den Registerbänden, die in der digitalen Präsentation eindeutig identifizierbar sind. Die Registereinträge weisen auf Bände der Protokolle oder Beilagen einer Legislaturperiode mit genauer Seitenzahl hin. Diese kann dann mit Hilfe der Paginierung in den digitalisierten Bänden aufgerufen werden.

In der Zuordnung des Volltextes und der weiterführenden Informationen sowie durch Akzentuierung einzelner Dokumente handelt es sich um ein kombiniertes Digitalisierungs- und Dokumentationsprojekt. Digitalisierung und Strukturdatenerschließung aufgrund von Vorgaben der Landesbibliothek wurden durch einen Dienstleister abgewickelt. Für die Projektabwicklung und Präsentation wird die Software GOOBI genutzt. Mit dem Projekt Landtagsprotokolle ist der WLB der Einstieg in die Massendigitalisierung und die Präsentation von Zeitschriftenbänden gelungen.

Christian Herrmann

\title{
Studienaufenthalt in Indonesien
}

Das umfassende Bestandsprofil der Bibelsammlung der Württembergischen Landesbibliothek führt zu Anfragen, aber auch längerfristigen Kontakten zu Personen und Institutionen weit über Deutschland hinaus. So besuchte der Generalsekretär der Indonesischen Bibelgesellschaft, Duta Pranowo, in Begleitung des als Dozent tätigen deutschen Missionars Dr. Christian Goßweiler im Juni 2012 die Landesbibliothek. Hauptgegenstand des Interesses war dabei eines von weltweit vier nachweisbaren Originalexemplaren des malaiischen Matthäusevangeliums von 1629 (Ba malai.1629 01). Es ist das älteste in einer asiatischen Sprache gedruckte und erhaltene Buch. Die indonesische Staatssprache ist eine Weiterentwicklung des Malaiischen. Ergebnis weiterer Beratung und Unterstützung (z.B. durch Übermittlung von Digitalisaten) ist eine Ende 2012 in Indonesien hergestellte Facsimile-Ausgabe sowie eine verbesserte Variante mitsamt Kommentarband in englischer und indonesischer Fassung. Zur offiziellen Präsentation der letzteren im Rahmen eines Symposiums in der Indonesischen Nationalbibliothek wurde ich als Referent in meiner Funktion als Leiter der Stuttgarter Bibelsammlung eingeladen. Am 23. April 2014 entfaltete ich in Jakarta Grundlinien der Geschichte der Missionsbibeln. Dr. Goßweiler zeichnete dann in diesen Zusammenhang die unterscheidenden Charakteristika und Entstehungsbedingungen der ältesten malaiischen Bibelausgabe ein. In der Aussprache ging es vor allem um Fragen der Kontextualisierung und Revision von Bibelübersetzungen. Die Übergabe zweier Dubletten im Vortrag erwähnter Missionsbibeln an die Indonesische Bibelgesellschaft sollte die Kooperationsbereitschaft der Württembergischen Landesbibliothek unterstreichen. 


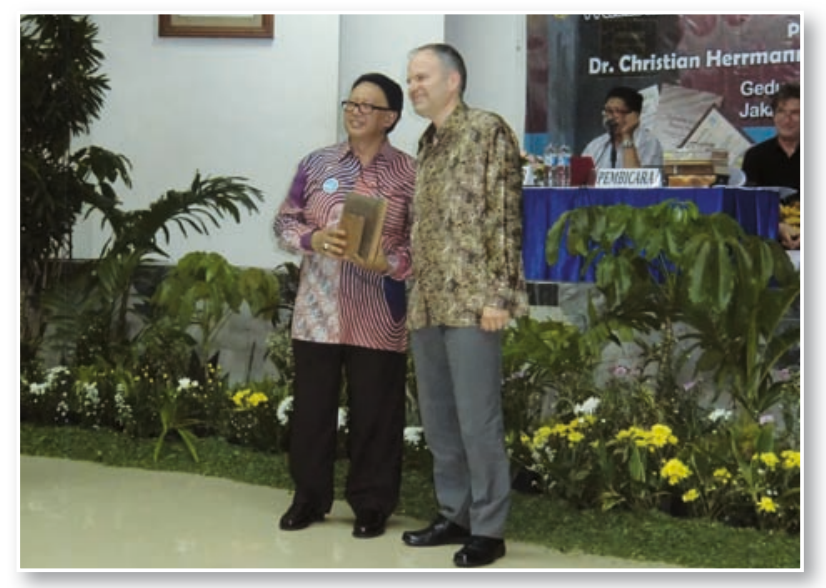

Abb.1: Dr. Christian Herrmann mit Duta Pranowo

Meine Frau und ich wurden zuvor durch die Abteilungen der Indonesischen Nationalbibliothek in Jakarta geführt. Von hier aus wird die bibliothekarische Arbeit in den Provinzen gesteuert, z.B. durch eine zentrale Datenverwaltung sowie Bücherlieferungen per Schiff. Datentechnisch sind die Südostasiaten ihren europäischen Kollegen in mancher Hinsicht voraus. Datenbanken, E-Books, ausgedehnte Lesebereiche mit Benutzer-PCs, Digitalisierungsprojekte, Facebook-Auftritt, elektronisches Gästebuch gehören seit längerem zum Service der Nationalbibliothek. Historische Fotos aus der Landes- und Bibliotheksgeschichte an Benutzer-Arbeitsplätzen und an der Informationstheke stehen für das Zueinander von Tradition und Moderne. In den historischen Sammlungen sind u.a. die Palmblatthandschriften hervorzuheben, außerdem alte Drucke aus der frühen Kolonialzeit sowie Fotoalben zur Landesgeschichte und seltenes Kartenmaterial. Die Lagerung ist insbesondere bei einer ehemals niederländischen Sammlung aus dem 19. Jahrhundert in konservatorischer Hinsicht bisher unbefrie-

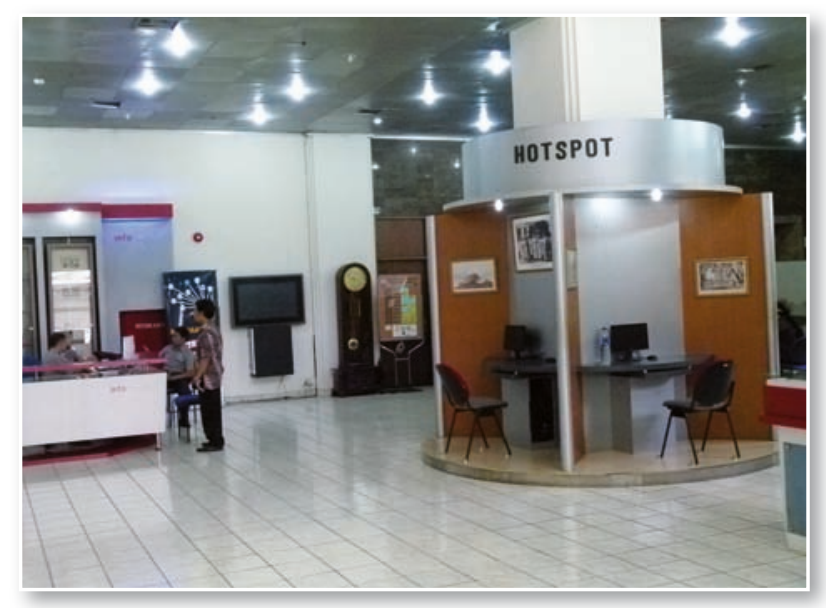

Abb. 2: Eingangsbereich der Indonesischen Nationalbibliothek digend gelöst. Europäische Sprachen wie Latein und Italienisch bzw. Deutsch und Niederländisch werden in den Titelaufnahmen manchmal ebenso verwechselt wie die christliche und islamische Zeitrechnung. Andererseits ist die Nationalbibliothek für eine Monographienreihe zur Geschichte südostasiatischer Handschriften bzw. zur Sprachentwicklung verantwortlich und verpflichtet ihre Mitarbeiter zu regelmäßigen wissenschaftlichen Publikationen. Der Öffentlichkeitsarbeit dienen zudem das Buchmuseum und der Vortragsraum mit 400 Sitzplätzen.

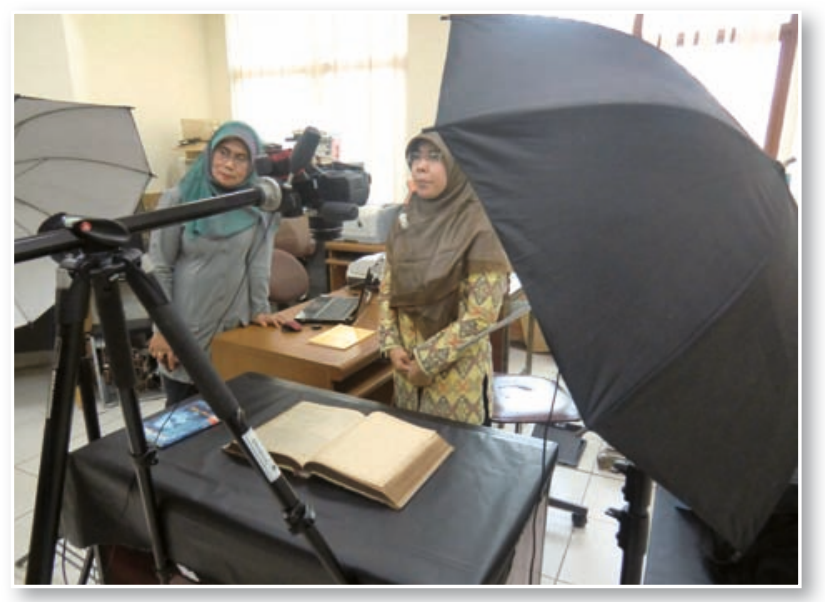

Abb. 3: Digitalisierungswerkstatt der Indonesischen Nationalbibliothek

Der zweite Tag der Studienreise wurde dazu genutzt, die Arbeit der Indonesischen Bibelgesellschaft näher kennen zu lernen. Weit gediehen sind Fundraising-Aktivitäten sowie die Kulturarbeit mit Ausstellungen, Vorträgen und Bibelmuseum (mit Exponaten zur Geschichte der Bibel und Bibelübersetzung). Exemplarisch konnten wir an der Eröffnung einer Ausstellung über Ikonen teilnehmen, zu der auch Vertreter orthodox geprägter Länder eingeladen waren. Die Bibliothek umfasst historische wie aktuelle Bibelausgaben vor allem in den für Indonesien relevanten Sprachen, aber auch in den biblischen Ursprachen sowie Hilfsmittel zum Bibelstudium. Die aktuellen Produkte der Bibelgesellschaft können in der angegliederten Buchhandlung erworben werden. Charakteristischerweise spricht ein Indonesier eine Stammes- bzw. Regionalsprache und zusätzlich die Staatssprache Bahasa Indonesia. Das Angebot an Bibeln ist entsprechend strukturiert. Englische Literatur wird mit Rücksicht auf die vorhandenen Sprachkenntnisse selten im Original gelesen, sondern meist übersetzt. Alle Gesprächspartner erwiesen sich als überaus freundlich und hilfsbereit. 


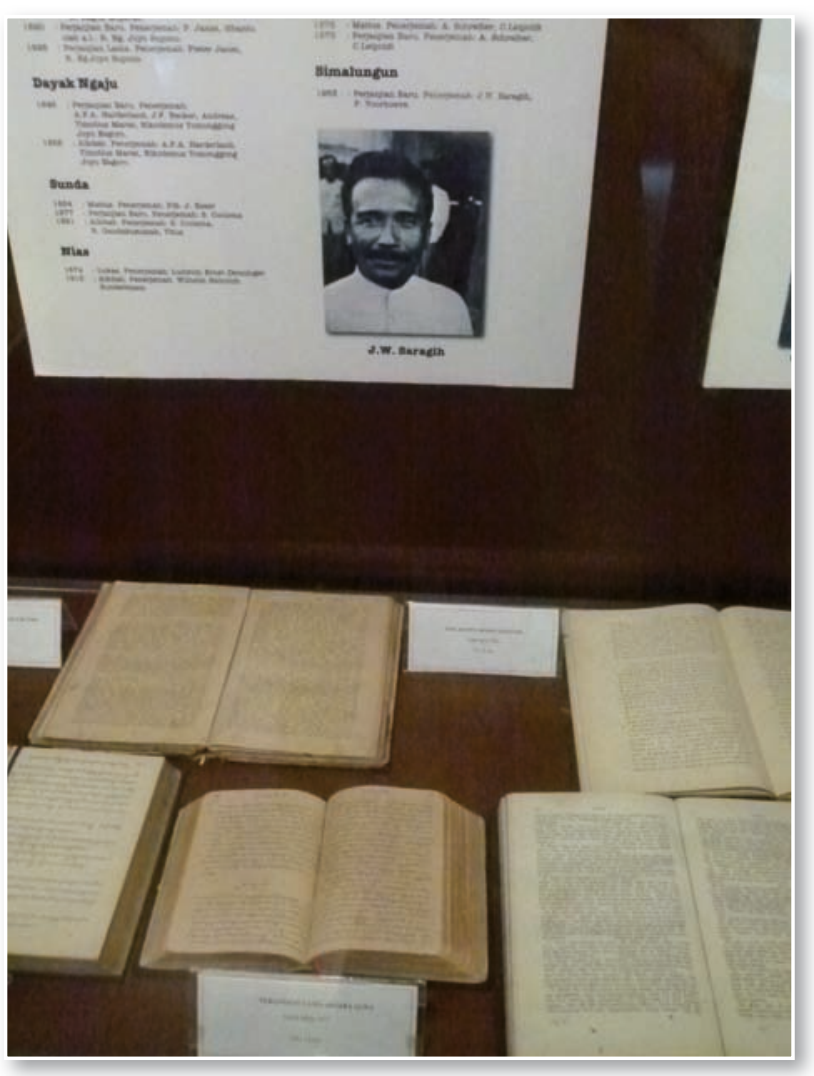

Abb. 4: Bibelmuseum der Indonesischen Bibelgesellschaft

Insgesamt bemüht man sich aufgrund der indonesischen Staatsphilosophie Pancasila um einen gesellschaftlichen Ausgleich von Gegensätzen sowie um Harmonie der Religionen unter dem Vorzeichen einer verbindlichen Zivilreligion. Im östlichen Teil Indonesiens funktioniert die Koexistenz der zugelassenen Religionen Islam, Christentum, Hinduis- mus und Buddhismus meist gut. In den anderen Regionen (z.B. Westjava, Süd-Sulawesi, Sumatra) kommt es hingegen immer wieder zur Verfolgung bzw. Diskriminierung von Christen. Wer die Zuordnung "Christ" im Personalausweis aufweist, kann dann z.B. bei der Vergabe von Arbeitsplätzen erhebliche Nachteile erfahren. Schwierig ist auch die Genehmigung von Kirchengebäuden und diakonischen Projekten sowie die Konversion ehemaliger Muslime. Der tatsächliche Anteil der Christen an der Bevölkerung liegt deutlich über den offiziellen $10 \%$. Besonders stark ist das Christentum unter der chinesischstämmigen Bevölkerung sowie bei bestimmten Stämmen (z.B. Batak, Dayak, Minahasa) vertreten. Die teilweise deutschen Missionare und Bibelübersetzer werden in einigen Regionen bis heute verehrt.

Über die Homepage der Württembergischen Landesbibliothek ist eine Übersicht zur Geschichte der Missionsbibeln, zu deren Übersetzern bzw. zu den verantwortlichen Institutionen (z.B. Missionsgesellschaften) zugänglich. Sie wird sukzessive mit den digitalen Volltextversionen der exemplarisch ausgewählten Bibelausgaben verlinkt. ${ }^{1}$

Christian Herrmann

(1) www.wlb-stuttgart.de/sammlungen/bibeln/bestand/missionsbibeln/

\section{Geschichte der frühen Missionsbibeln}

\section{Was ist eine "Missionsbibel“?}

"So kommt der Glaube aus der Predigt, das Predigen aber durch das Wort Christi" (Römer 10,17). Glaube entsteht durch die Wirkung des Wortes Gottes. Das Wort Gottes wird offenbart, begegnet zunächst mündlich in der biblischen Heilsgeschichte, dann in schriftlicher Form. Was Wort Gottes ist, lässt sich konkret und verbindlich festmachen an dem, was in der Bibel steht. Der Heilige Geist bindet sich so an die Heilige Schrift, dass sie soweit es Gott gefällt - bei dem ihrer Kraft ausgesetzten Menschen in doppelter Weise Glauben bewirkt. ${ }^{2}$ Es geht um den Glaubensakt im Sinne einer lebendigen Beziehung zu Gott und um den Glaubensinhalt. Die Bibel allgemein ist zwar auch eine historische Schrift mit einem bestimmten räumlichen, sprachlichen und zeitlichen Kontext - wie andere antike Dokumente auch. Aber sie weist wesensmäßig auch einen heilsgeschichtlichexistenziellen Kontext auf, entfaltet eine Dynamik eigener Art. Jede Bibel ist im weiteren Sinne eine Missionsbibel, weil sie auf die Entstehung, Festigung, Vertiefung von Glauben ausgerichtet ist. Weil es durch das Zueinander von Glaubensakt

(1) Leicht modifizierte deutsche Langfassung eines in englischer Sprache gehaltenen Vortrags (23.4.2014, Indonesische Nationalbibliothek, Jakarta). Die Belegbände werden z.T. nur zitiert, nicht mit Bild gezeigt.

(2) Vgl. Augsburgisches Bekenntnis, Artikel V. 